\title{
Detailed kinematics and morphological features in tidally heated disks
}

\author{
C. Mastropietro ${ }^{1}$, B. Moore $^{1}$, L. Mayer ${ }^{1}$, V. P. Debattista ${ }^{2,3}$, \\ R. Piffaretti ${ }^{1,4}$ and J. Stadel ${ }^{1}$ \\ ${ }^{1}$ Institute for Theoretical Physics, University of Zürich, CH-8057 Zürich, Switzerland \\ ${ }^{2}$ Astronomy Dept., University of Washington Seattle WA 98195-1580 \\ ${ }^{3}$ Institut für Astronomie, ETH Zürich, CH-8093 Zürich, Switzerland \\ ${ }^{4}$ Institut für Astrophysik, Leopold-Franzens Universität Innsbruck, A-6020 Innsbruck, Austria

\begin{abstract}
The recent discovery of hidden non-axysimmetric and disk-like structures in bright Virgo dwarf elliptical and lenticular galaxies (dE/dSph/dS0) indicates that they may have latetype progenitors. Using N-body simulations we follow the evolution of disk galaxies within a $\Lambda \mathrm{CDM}$ cluster simulated with $10^{7}$ particles, where the hierarchical growth and galaxy harassment are modeled self-consistently. Most of the galaxies undergo significant morphological transformation, even at the outskirts of the cluster, and move through the Hubble sequence from late type disks to dwarf spheroidals. The time evolution of the simulated galaxies is compared with unsharp-masked images obtained from VLT data and the projected kinematics of our models with the latest high resolution spectroscopic studies from the Keck and Palomar telescopes.
\end{abstract}

Keywords. methods: N-body simulations - galaxies: clusters: general - galaxies: evolution

\section{Introduction}

Early type dwarfs ( $\mathrm{dE}$ and $\mathrm{dSO}$ ) are the most common type of galaxies in the nearby universe, yet their origin and nature are still unknown. These galaxies are found nearly exclusively near bright galaxies and in groups or clusters. Formation scenarios include mainly two different hypotheses: 1) dEs and dSOs are primordial galaxies, 2) they are the result of a morphological transformation of spiral and irregular galaxies accreting into the cluster. Moore, Lake \& Katz (1998) have shown that galaxy harassment in clusters can transform spirals into spheroidals. According to this evolutionary scenario we expect to find nearby cluster galaxies that are currently undergoing morphological transformation and retain part of their disk nature. Recent observations confirm that early type galaxies have a broad range of photometric and kinematical characteristics. Jerjen, Kalnajs \& Binggeli (2000) and Barazza, Binggeli \& Jerjen (2002) discovered hidden spiral structures and bar features in bright dEs in the Virgo cluster. Spirals and disks were also observed within dwarf spheroidal galaxies in the Coma (Graham, Jerjen \& Guzmán 2003 and Fornax clusters (De Rijcke et al. 2001). Furthermore, the degree of rotational support is found to vary from zero to a value close to one expected for a galaxy flattened by rotation (De Rijcke et al. 2001; Simien \& Prugniel 2002; Pedraz et al. 2002; Geha, Guhathakurta \& van der Marel 2002; Geha, Guhathakurta \& van der Marel 2003; van Zee, Skillman \& Haynes 2004). The aim of this work is to follow the evolution of disk galaxies orbiting in a cluster environment using high resolution N-body simulations and to compare the final harassed remnants with the latest photometric and spectroscopic data. 


\begin{tabular}{|c|c|c|c|c|c|c|c|c|c|c|c|c|c|c|c|}
\hline Gal & Apo-Pe & $r_{t}$ & $r_{e}$ & $M_{h}$ & $M_{s}$ & $b / a$ & $c / a$ & Gal & Ap & $r_{t}$ & $r_{e}$ & $M_{h}$ & $M_{s}$ & $|b / a|$ & $\mid c / a$ \\
\hline G1 & $200-90$ & 8.5 & 2.0 & 2.6 & 2.3 & 0.79 & $\mid 0.39$ & G11 & -460 & $\mid 17.2$ & $\mid 2.0$ & 8.2 & 3.6 & | $0.71 \mid$ & $\mid 0.18$ \\
\hline G2 & $150-100$ & 10.1 & 1.3 & 2.8 & 2.4 & 0.57 & 0.40 & G12 & $1130-120$ & 16.9 & 2.6 & 14.6 & 4.1 & 0.66 & 0.18 \\
\hline G3 & $650-60$ & 16.6 & 2.7 & 13.0 & 4.2 & 0.42 & 0.18 & G13 & $\ldots-900$ & 17.4 & 2.6 & 19.2 & 4.2 & 0.41 & 0.28 \\
\hline G4 & $300-50$ & 11.6 & 1.8 & 5.2 & 3.2 & 0.44 & 0.34 & G14 & $\ldots-\ldots$ & 16.3 & 2.5 & 24.4 & 4.2 & 0.79 & 0.29 \\
\hline G5 & $630-100$ & 19.4 & 1.9 & 7.6 & 3.3 & 0.60 & 0.27 & G15 & $2480-$. & 17.0 & 2.9 & 21.1 & 4.2 & 0.43 & 0.19 \\
\hline G6 & $90-40$ & 3.6 & 0.7 & 0.2 & 0.5 & 0.81 & 0.80 & G16 & $720-210$ & 14.7 & 2.6 & 18.8 & 4.1 & 0.45 & 0.23 \\
\hline G7 & $75-210$ & 11.0 & 1.8 & 4.2 & 3.3 & 0.61 & 0.38 & G17 & $\ldots-\ldots$ & 15.6 & 2.6 & 21.0 & 4.2 & 0.38 & 0.22 \\
\hline G8 & $100-60$ & 4.6 & 1.1 & 1.0 & 1.5 & 0.87 & 0.86 & G18 & 2290-. & 15.2 & 2.7 & 19.5 & 4.2 & 0.41 & 0.25 \\
\hline G9 & $120-70$ & 7.9 & 1.1 & 1.5 & 1.8 & 0.66 & 0.54 & G19 & $2810-\ldots$ & 15.0 & 3.0 & 19.6 & 4.2 & 0.77 & 0.17 \\
\hline G10 & $270-80$ & 9.0 & 1.2 & 1.8 & 1.8 & 0.62 & 0.49 & G20 & $1720-450$ & 16.8 & 2.9 & $\mid 21.6$ & 4.2 & $|0.34|$ & 0.22 \\
\hline
\end{tabular}

Table 1. Final state of the remnants. The second column indicates, when available, the apocentric and pericentric distances of the last orbit. For each galaxy we list three dimensional tidal $r_{t}$ and effective $r_{e}$ radius (in kpc), dark matter $M_{h}$ and stellar $M_{s}$ mass (in units of $10^{9} M_{\odot}$ ). The last two columns indicate the axial ratios (respectively intermediate and short axis to major axis) measured within $2 r_{e}$.

\section{Galaxy evolution}

All the simulations have been carried out using PKDGRAV (Stadel 2001). The galaxy model is a multi-component system with an exponential stellar disk embedded in a NFW (Navarro, Frenk \& White 1997) spherical dark matter halo and was constructed using the technique described by Hernquist (1993). The structural parameters of the disk and the halo are chosen so that the resulting rotation curve resembles that of a typical bulgeless late-type $(\mathrm{Sc} / \mathrm{Sd}$ ) disk galaxy. The mass within the virial radius was set equal to $7 \times 10^{10} \mathrm{M}_{\odot}$ and the fraction of mass in the disk is $\sim 6 \%$. The model has a central surface brightness $\mu_{B}=22 \mathrm{mag} \operatorname{arcsec}^{-2}$. Each galaxy is modeled with $10^{6}$ particles, $10^{5}$ of which are in the disk. The galaxies were evolved for $\sim 8.4$ Gyrs in a $\Lambda$ CDM cluster with a mass at $z=0$ comparable to that of Virgo $\left(M_{v i r}=3.1 \times 10^{14} \mathrm{M}_{\odot}\right)$ and a virial radius $R_{v i r}$ of $1.8 \mathrm{Mpc}$ (Diemand, Moore \& Stadel 2004). The number of particles within the virial radius is $\sim 6 \times 10^{6}$.

The evolution of many of the galaxies within the cluster is quite violent and due to a sequence of strong gravitational encounters with substructures and with the global cluster potential. Most of the central galaxies lose a significant fraction of stars and undergo a complete morphological transformation from disks to spheroidal systems (Fig. 1, left panel). Depending on the orbit and on the number of close encounters that the galaxy suffers, the morphological transformation of the stellar component can be complete and produce a pure spheroidal system, or can lead to the formation of a more elongated structure, which still retains disk and bar features. For a few galaxies orbiting closest to the cluster center the tidal effects from the cluster potential become dominant and the evolution is very rapid. Table 1 summarises the main properties of the remnant galaxies. The initial model has a virial radius of $85 \mathrm{kpc}$ and the effective radius of the stellar distribution is $2.5 \mathrm{kpc}$. In a few cases the galaxy loses much more dark matter than stars and becomes baryon dominated in the central region. The loss of stellar mass is not a simple function of the mean orbital radius or apocentric/pericentric distance from the cluster center, confirming the importance of harassment. There is a clear correlation between the flattening of the stellar component (c/a) and the final stellar mass: massive stellar remnants have smaller c/a values and retain more of their initial disk nature, while galaxies that have lost most of their stars tend to be more spheroidal. 

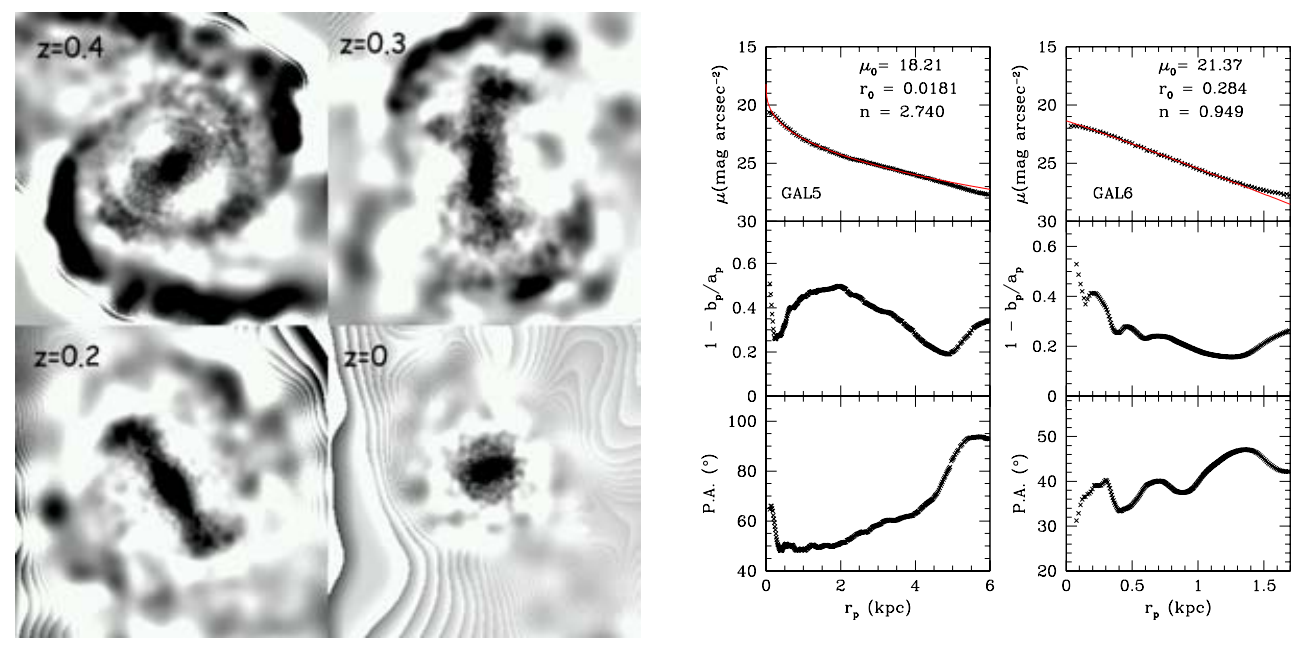

Figure 1. Left panel: morphological transformation of a disk galaxy into a dwarf elliptical. Right panel: $B$-band surface brightness, ellipticity and position angle profile for two galaxies of our sample. The coordinate $r_{p}$ is the equivalent radius. A Sérsic law is superimposed on the surface brightness profile (red solid line) and the corresponding best fit parameters are given in the top right of the panel. Note that $r_{0}$ is in $\mathrm{kpc}$ units.

\section{Photometric analysis and kinematics}

In order to compare the results of our simulations with observations, we projected the remnants along a random line of sight and calculated surface brightness, ellipticity and position angle profiles (see Fig. 2, right panel). Even though there is not a well defined trend in the ellipticity profiles, galaxies which preserve a bar component are generally characterised by large ellipticities in the central $2-3 \mathrm{kpc}$ and by a drop in the outer regions. Often the inner $0.5 \mathrm{kpc}$ exhibits a very low ellipticity corresponding to a small nuclear component produced by buckling instabilities, whereas the external drop is more likely due to disk heating. The surface brightness profiles were fitted with a Sérsic law. The range of surface brightness parameters agrees quite well with the observations of dwarf galaxies in Virgo.

We measure the kinematics of the our projected galaxies using a slit lenght which allows us to observe in most of the galaxies the turnover of the rotation curve. In most of the cases the remnants lose a large fraction of rotation velocity (see Fig. 2, left panel), but only for one galaxy (GAL6) we do not observe any significative rotation along the major axis. In general we find non-rotating and slowly rotating dwarfs to have internal and environmental properties substantially different from the rotating ones. In particular the former are relatively low massive objects with high c/a ratios and less apparent disk features. They seem to be the product of a violent morphological transformation accelerated by the proximity of the cluster center. Although the galaxies lose significant amounts of mass, the velocity dispersion can rise up to $50 \mathrm{~km} \mathrm{~s}^{-1}$ in the central 1-2 kpc. This is due to the bar formation and the transformation of circular to more radial orbits. The velocity dispersion profiles are typically decreasing at larger radii but they rise again in those galaxies for which the slit semi-length approaches or exceeds the tidal radius. As the ratio between rotational velocity and velocity dispersion decreases, the galaxy loses rotational support and the flattening becomes dominated by anisotropic pressure. We plotted in Fig. 2 (right panel) the ratio of the maximum rotational velocity $v_{\max }$ to the mean velocity dispersion $\bar{\sigma}$ within a radius of $6 \mathrm{kpc}$ versus the mean isophotal ellipticity 

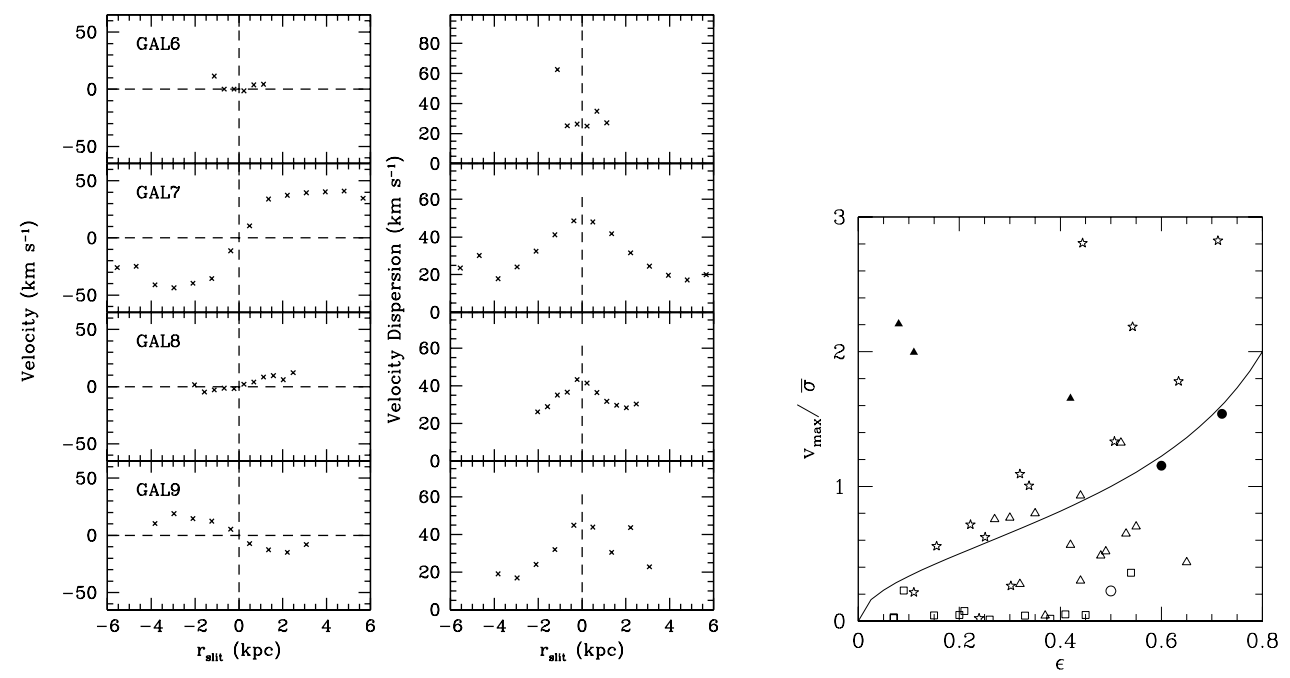

Figure 2. Left panel: projected kinematic profiles of the remnants within the cluster virial radius at $z=0$. The line of sight velocity (left panels) and the velocity dispersion (right panels) are plotted as a function of the radial distance along the major axis. Right panel: the ratio of the maximum rotational velocity $v_{\max }$ to the mean velocity dispersion $\bar{\sigma}$ plotted versus the mean isophotal ellipticity. Stars represent the simulated sample, while the other points are observational data.

calculated in the same radial range. In the same plot we show the observational results for most of the dwarf galaxies in Virgo with published kinematics. We also plot the data for dwarf galaxies in the Fornax cluster by De Rijcke et al. (2003). Most of the simulated remnants have a similar range of ellipticities and $v_{\text {max }} / \bar{\sigma}$ as the observed dwarfs, even if it is clear that we have some difficulties in reproducing galaxies with a degree of rotational support close to zero. On the other hand it is possible that the low $v_{\text {max }} / \bar{\sigma}$ ratio of some galaxies is due to the small radial range covered by the observations.

\section{References}

Barazza, F.D., Binggeli, B. \& Jerjen, H. 2002, A\& $A$ 391, 823

Bender, R. \& Nieto, J.L. 1990, A\&\&A 239, 97

Conselice, C.J., Gallagher, J.S. \& Wyse, R.F.G. 2001, ApJ 559, 791

De Rijcke, S., Dejonghe, H., Zeilinger, W.W., \& Hau, G.K.T. 2001, ApJ 559, L21

De Rijcke, S., Dejonghe, H., Zeilinger, W.W. \& Hau, G.K.T. 2003, A\&A 400, 119

Diemand, J., Moore, B. \& Stadel, J. 2004, MNRAS 353, 624

Geha, M., Guhathakurta, P. \& van der Marel, R.P. 2002, AJ 124, 3073

Geha, M., Guhathakurta, P. \& van der Marel, R.P. 2003, AJ 126, 1794

Graham, A.W., Jerjen, H. \& Guzmán, R. 2003, AJ 126, 1787

Hernquist, L. 1993, ApJ 86, 389

Jerjen, H., Kalnajs, A. \& Binggeli, B. 2000, A\& A 358, 845

Moore, B., Lake, G. \& Katz, N. 1998, ApJ 495, 139

Navarro J.F., Frenk C.S. \& White S.D.M. 1997, ApJ, 490, 493

Pedraz, S., Gorgas, J., Cardiel, N., Sánchez-Blázquez, P. \& Guzmán, R. 2002, MNRAS 332, L59

Simien, F. \& Prugniel, P. 2002, A $\mathscr{E} A$ 384, 371

Stadel, J. 2001, Ph.D. Thesis

van Zee, L., Skillman, E. D. \& Haynes, M. P. 2004, AJ 128, 121 


\section{Discussion}

CONSELICE: How much stellar mass is lost in these simulated disks?

Mastropietro: Up to $90 \%$.

CALDWELL: The models you created that are not purely exponential have support in the real universe, because in nearby clusters the more massive dwarf ellipticals are more centrally concentrated than pure exponentials, and look very much like your models. Also, in addition to the weak disk structure seen in some dwarf ellipticals, many have ages of around $2-3$ Gyr. Thus if we image clusters at $\mathrm{z} \approx 0.2$, we should see many more dwarfs with disk-like structures shown in your models.

Mastropietro: Yes, most of them should appear like disk galaxies at $\mathrm{z} \approx 0.4$.

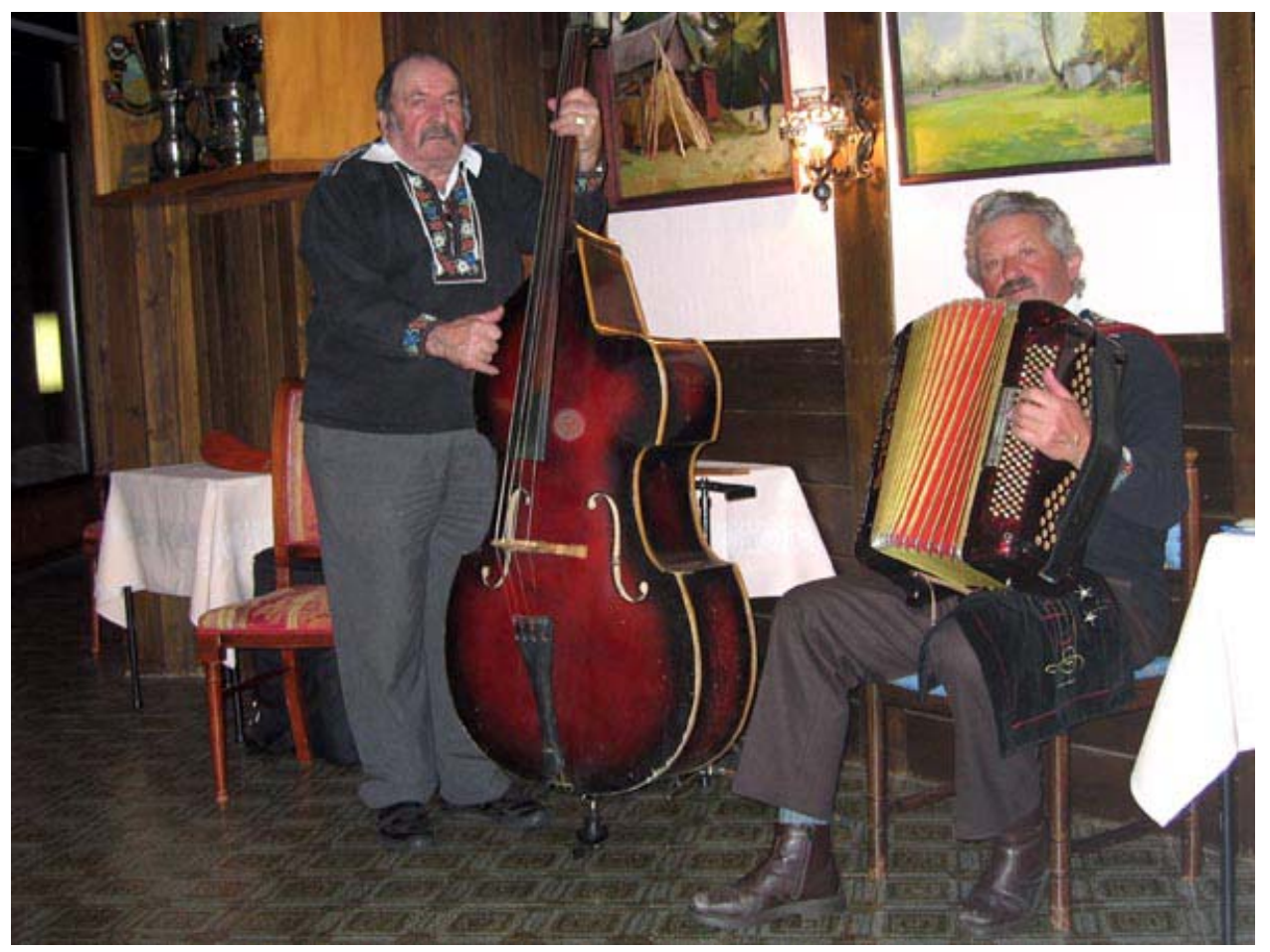

